\title{
Thin film dielectric microstrip kinetic inductance detectors
}

\author{
Benjamin A. Mazin, ${ }^{1, a)}$ Daniel Sank, ${ }^{1}$ Sean McHugh, ${ }^{1}$ Erik A. Lucero, ${ }^{1}$ \\ Andrew Merrill, ${ }^{1}$ Jiansong Gao, ${ }^{2}$ David Pappas, ${ }^{2}$ David Moore, ${ }^{3}$ and Jonas Zmuidzinas ${ }^{3}$ \\ ${ }^{1}$ Department of Physics, University of California-Santa Barbara, Santa Barbara, \\ California 93106-9530, USA \\ ${ }^{2}$ National Institute of Standards and Technology, Boulder, Colorado 80305-3328, USA \\ ${ }^{3}$ Department of Physics, California Institute of Technology, Pasadena, California 91125, USA
}

(Received 7 October 2009; accepted 21 January 2010; published online 8 March 2010)

\begin{abstract}
Microwave kinetic inductance detectors, or MKIDs, are a type of low temperature detector that exhibit intrinsic frequency domain multiplexing at microwave frequencies. We present the first theory and measurements on a MKID based on a microstrip transmission line resonator. A complete characterization of the dielectric loss and noise properties of these resonators is performed, and agrees well with the derived theory. A competitive noise equivalent power of $5 \times 10^{-17} \mathrm{~W} \mathrm{~Hz}^{-1 / 2}$ at $10 \mathrm{~Hz}$ has been demonstrated. The resonators exhibit the highest quality factors known in a microstrip resonator with a deposited thin film dielectric. () 2010 American Institute of Physics. [doi:10.1063/1.3314281]
\end{abstract}

Thin film superconducting microwave resonators have been an area of intense research in the past decade for microwave kinetic inductance detectors (MKIDs) (Ref. 1) for submillimeter, ${ }^{2}$ optical/UV, ${ }^{3}$ and $\mathrm{x}$-ray ${ }^{4}$ astrophysics, components in superconducting qubits, ${ }^{5,6}$ and fundamental studies in condensed matter physics such as searches for macroscopic quantum states ${ }^{7}$ and measurements of the properties of cryogenic liquids. ${ }^{8}$ These resonators have primarily been coplanar waveguide (CPW) transmission line resonators. ${ }^{9} \mathrm{~A}$ CPW transmission line is a planar structure with a center strip and slots that separate the center strip from ground planes on either side, as shown in the right panel of Fig. 1. They are simple to fabricate out of a single superconducting film on a crystalline dielectric. However, in many cases the flexibility of a microstrip resonator, which is a stacked structure with a deposited dielectric separating two conductors, would be advantageous. A microstrip MKID can be deposited on any material, instead of just single crystal silicon or sapphire, and can be made significantly smaller than a CPW MKID. This allows more flexibility in detector design. The low loss deposited dielectrics required for a sensitive microstrip MKID have many other uses, such as in the lumped element capacitors and wiring crossovers in superconducting qubits ${ }^{10}$ or the microstrip combiner networks of planar antenna arrays. ${ }^{11}$ This work details the first microstrip MKID using a deposited thin film dielectric.

Microstrip resonators can be made very sensitive by making the dielectric thickness comparable to or thinner than the penetration depth of the superconductor; since this causes the device to be dominated by the kinetic inductance of the superconductor, not the magnetic inductance of the transmission line. This leads to a very sensitive detector since MKIDs respond to changes in the kinetic inductance, such as those caused by broken Cooper Pairs from photon absorption. It also makes a compact resonator since the phase velocity on the transmission line can be lower than several percent of the speed of light. Following Swihart ${ }^{12}$ and Pond et al., ${ }^{13}$ the phase velocity of a superconducting microstrip whose width $w$ is much greater than the dielectric thickness $d$ and at $T \ll T_{c}$ can be written using the London equation as

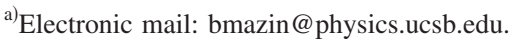

$$
v_{p}=c\left\{\epsilon_{r}\left[1+\frac{\lambda_{1}}{d} \operatorname{coth}\left(\frac{t_{1}}{\lambda_{1}}\right)+\frac{\lambda_{2}}{d} \operatorname{coth}\left(\frac{t_{2}}{\lambda_{2}}\right)\right]\right\}^{-1 / 2},
$$

where $c$ is the speed of light in vacuum, $\epsilon_{r}$ is the relative dielectric constant of the microstrip dielectric, and $\lambda$ and $t$ are the penetration depth and the thickness of the top (1) and bottom (2) superconductors. The kinetic inductance fraction, $\alpha=L_{k} / L_{T}$, is defined as the ratio of the kinetic inductance $L_{k}$ to the total inductance, $L_{T}=L_{k}+L_{m}$, where $L_{m}$ is the magnetic inductance of the transmission line. Using Eq. (1) and the phase velocity of a perfectly conducting transmission line $v_{p N}=c / \sqrt{\epsilon_{r}}$ we can calculate $\alpha$ for a uniform distribution of quasiparticles in both the top and bottom microstrip wiring as follows:

$\alpha=1-\left(\frac{v_{p}}{v_{p N}}\right)^{2}=1-\left[1+\frac{\lambda_{1}}{d} \operatorname{coth}\left(\frac{t_{1}}{\lambda_{1}}\right)+\frac{\lambda_{2}}{d} \operatorname{coth}\left(\frac{t_{2}}{\lambda_{2}}\right)\right]^{-1}$.

The microstrip MKID is read out by sending a microwave probe signal past the resonator, and a homodyne mixing scheme is used to recover the phase and amplitude

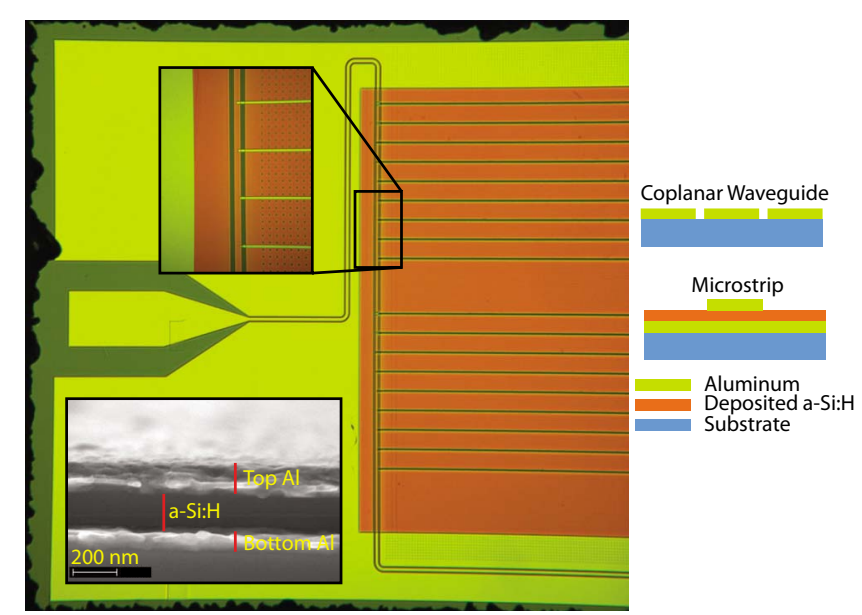

FIG. 1. (Color online) Right: A cross-sectional view of a conventional CPW transmission line and the microstrip transmission lines used in this paper. Left: An optical microscope image of a portion of the tested device. The inset on the lower left shows a SEM image of the cross section of a device that has been cleaved. 
changes imprinted onto the carrier by the MKID. ${ }^{1}$ Using Eqs. (2) and (3), ${ }^{14}$ we can express the expected responsivity, normalized so the microwave transmission past the resonator $S_{21}$ far off resonance is unity, of a microstrip MKID in both amplitude and phase as

$$
\frac{\partial S_{21}}{\partial N_{\mathrm{qp}}}=\frac{\alpha|\gamma| \kappa Q_{m}^{2}}{V Q_{c}}
$$

with

$$
\kappa \approx \frac{1}{\pi N_{0}} \sqrt{\frac{2}{\pi k T \Delta_{0}}} \sinh (\xi) K_{0}(\xi)+j \frac{1}{2 N_{0} \Delta_{0}}\left[1+\sqrt{\frac{2 \Delta_{0}}{\pi k T}} e^{-\xi} I_{0}(\xi)\right]
$$

where $N_{\mathrm{qp}}$ is the number of quasiparticles in the resonator, $Q_{m}$ is the measured quality factor, $Q_{c}$ is the coupling quality factor, $V$ is twice the volume of the top microstrip wiring layer since this is where the current flows and where quasiparticles effectively contribute to the surface impedance, $N_{0}$ is the single spin density of states, $\Delta_{0}$ is the effective gap at $T \approx 0, \xi=\hbar \omega / 2 k T$, and $\gamma$ is constant that varies from $-1 / 3$ in the extreme anomalous limit to -1 in the thin film local limit. The predicted phase responsivity in radians per quasiparticle, $\partial \theta / \partial N_{\mathrm{qp}}$, can be found by taking the imaginary part of Eq. (3) and dividing by the radius of the resonance loop, $Q_{m} / 2 Q_{c}$, while the dissipation response $\partial D / \partial N_{\mathrm{qp}}$ is found by taking the real part and dividing by the radius of the resonance loop. $Q_{m}$ is related to $Q_{c}$ and the quality factor resulting from any source of dissipation in the system, $Q_{i}$, by the relation $Q_{m}^{-1}=Q_{c}^{-1}+Q_{i}^{-1}$. We operate the devices in this paper at $T<T_{c} / 8$, so there are essentially no thermal quasiparticles in the devices. At these temperatures and in a device in which there is no trapped magnetic flux, $Q_{i}$ should be dominated by losses in the microstrip dielectric. This expression for the responsivity of a microstrip MKID can be combined with the known amplifier noise, providing a closed form expression that can predict the amplifier noise limited sensitivity of the MKID at a given readout power level.

Previous work with lumped element resonators ${ }^{15}$ has shown that hydrogen rich amorphous silicon $(\mathrm{a}-\mathrm{Si}: \mathrm{H})$ is a promising material, with a low power loss $\operatorname{tangent} \tan (\delta)$ $\sim 2 \times 10^{-5}$. A low loss tangent, and hence a higher internal quality factor since $\tan (\delta)=1 / Q_{i}$, is vital for microstrip MKIDs. A higher $Q_{i}$ will directly lead to resonators with higher sensitivity when using a readout scheme based on dissipation. Because two-level system (TLS) fluctuators in the deposited amorphous dielectric produce frequency noise, ${ }^{16,17}$ it is likely that dissipation readout of the resonator response will be more sensitive. A higher $Q_{i}$ (and therefore $Q_{m}$ ) will directly lead to a more sensitive detector.

In this work, microstrip resonators with aluminum wiring and an a-Si:H dielectric have been fabricated. The geometry is illustrated in Fig. 1. The resonators were fabricated with optical lithography and dry etching techniques. First, a $93 \mathrm{~nm}$ thick aluminum film is deposited to form a CPW feed line and a ground plane with small ground plane holes to suppress effects from stray magnetic fields. This layer is then patterned with a dry etch in an inductively coupled plasma (ICP) etcher. A $200 \mathrm{~nm}$ thick film of a-Si:H is then deposited at a temperature of $100 \mathrm{C}$ to form the insulating dielectric layer. A $154 \mathrm{~nm}$ thick layer of aluminum is then sputtered. This layer is patterned by an ICP to form the top conductor of the microstrip. This forms microstrip resonators with a width $w$ of $4 \mu \mathrm{m}$ and lengths between 3.9 and $6.0 \mathrm{~mm}$. The

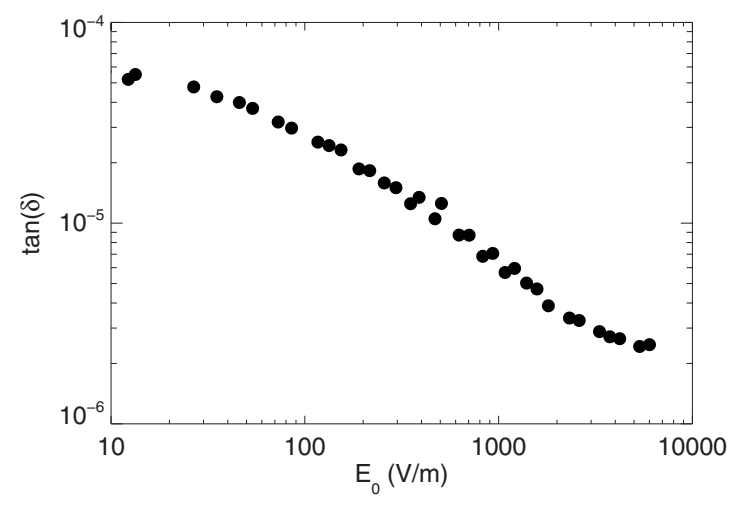

FIG. 2. The loss tangent of the a-Si:H dielectric, $\tan (\delta)$, dramatically decreases as the electric field in the resonator increases. The electric field $E_{0}$ shown is the value at the open ends of the half wave resonator tested. This resonator is $3985 \mu \mathrm{m}$ long, with $Q_{m}=35500$ and a resonant frequency of $9.054 \mathrm{GHz}$ when operated under an optimal magnetic field of $30 \mathrm{mG}$. It shows a fractional frequency noise (Ref. 17) of $4.1 \times 10^{-18} \mathrm{~Hz}^{-1}$ at a readout power of $-91 \mathrm{dBm}$ (equivalent to a current density of $17.5 \mathrm{~A} / \mathrm{m}$ ).

strength of the coupling $\left(Q_{c}\right)$ of the microstrip to the feedline is determined by the amount of microstrip line that covers the CPW feedline. Finally, the a-Si:H insulating layer is patterned with the ICP to allow easy wire bonding, and the silicon wafer is diced into $7.5 \times 2 \mathrm{~mm}$ chips.

The chips are glued into a gold-plated copper sample box with VGE-7031 varnish and wire bonded to transition boards which convert from the coaxial input lines to a CPW transmission line. The box is placed inside an adiabatic demagnetization refrigerator (ADR) capable of reaching base temperatures below $100 \mathrm{mK}$. A coaxial feedline drives the device through a $30 \mathrm{~dB}$ attenuator at 4 Kelvin, and a high electron mobility (HEMT) amplifier with a noise temperature $T_{n} \approx 5.5 \mathrm{~K}$ is used to boost the output signal. A cryoperm magnetic shield is used to shield the device, and a Helmholtz coil internal to the magnetic shield is used to apply a magnetic field normal to the surface of the chip.

Microstrip resonators, like CPW resonators, ${ }^{18}$ appear to be quite sensitive to the magnetic field normal to the metal surface during cooling through the superconducting transition temperature $T_{c}$. Despite the magnetic shield the best quality factor is achieved with an applied magnetic field of around $30 \mathrm{mG}$, most likely needed to cancel stray fields from the ADR magnet. Once cold, the devices showed little response to applied magnetic fields of over several hundred milligauss, where the resonator $Q_{i}$ starts degrading. This degradation remains when the field is ramped down to zero. When the device was warmed past $T_{c}$ and recooled the original $Q_{i}$ was recovered, indicating trapped magnetic flux.

Using the measured thickness of the a-Si:H film of 200 $\mathrm{nm}$ from Fig. 1 and $50 \mathrm{~nm}$ as the penetration depth of aluminum, Eq. (1), and the known lengths and resonant frequencies of the resonators it is possible to derive the dielectric constant of a-Si:H. This calculation yields $\epsilon_{r}=11.4$.

Previous work has shown that the dielectric loss tangent is strongly dependent on the electric field in the dielectric. ${ }^{6,15-17}$ Figure 2 shows the loss tangent as a function of electric field in one of the a-Si:H microstrip resonators. The electric field at the open ends of the resonator can be calculated from ${ }^{14}$ 


$$
E_{0}=\frac{1}{d} \sqrt{\frac{4 Z_{0}}{\pi m} \frac{Q_{m}^{2}}{Q_{c}} P}
$$

where $m=1 / 2$ for a half wave resonator, $P$ is the microwave power on the feedline, and $Z_{0}$ is the characteristic impedance of the microstrip transmission line $Z_{0}=\sqrt{L / C}=1 / v_{p} C$. Since $d \ll w$ we use a parallel plate capacitor approximation for the capacitance per unit length, $C=\epsilon_{0} \epsilon_{r} w / d$.

Since resonators used as detectors will nearly always be operated at the highest readout power possible before nonlinear effects set in, the right side of the plot with loss tangents below $2 \times 10^{-6}$ are the most relevant for MKIDs. The flattening of the loss tangent toward the right side of the plot is most likely due to the high readout power generating quasiparticles in the resonator.

The sensitivity of the MKID can be calculated by first taking noise spectra on resonance in both the phase $\left(S_{\theta}\right)$ and dissipation $\left(S_{D}\right)$ direction at a readout power just below the level where the MKID becomes nonlinear. The dimensionless phase or dissipation shift per quasiparticle referenced to the center of the resonance loop, $\partial \theta / \partial N_{\mathrm{qp}}$ and $\partial D / \partial N_{\mathrm{qp}}$, can be computed by taking a temperature sweep of the resonance loop and converting the temperature to an effective number of quasiparticles in the resonator center strip, $N_{\mathrm{qp}}$ $=2 N_{0} V \sqrt{2 \pi k_{B} T \Delta_{0}} e^{-\Delta_{0} / k_{B} T}$. Performing this operation on the resonator measured in Fig. 2 leads to $\partial \theta / \partial N_{\mathrm{qp}}=8.2 \times 10^{-7}$ and $\partial D / \partial N_{\mathrm{qp}}=3.8 \times 10^{-7}$ radians per quasiparticle. Equation (3) predicts $\partial \theta / \partial N_{\mathrm{qp}}=4.9 \times 10^{-7}$ and $\partial D / \partial N_{\mathrm{qp}}=1.3 \times 10^{-7}$. The match between the predicted and measured responsivities is reasonably good, showing that the derived responsivity formalism is a reasonably good approximation.

Using the measured noise spectra with this responsivity and a conservative quasiparticle lifetime $\tau_{\mathrm{qp}}$ in aluminum of $250 \mu$ s allows us to calculate the $\operatorname{NEP}_{\theta},{ }^{9}$

$\operatorname{NEP}_{\theta}^{2}(\omega)=S_{\theta}(\omega)\left(\frac{\eta \tau_{\mathrm{qp}}}{\Delta_{0}} \frac{\partial \theta}{\partial N_{\mathrm{qp}}}\right)^{-2}\left(1+\omega^{2} \tau_{\mathrm{qp}}^{2}\right)\left(1+\omega^{2} \tau_{\mathrm{res}}^{2}\right)$,

where $\tau_{\text {res }}=Q_{m} / \pi f_{0}$. This same equation can be used to calculate $\mathrm{NEP}_{D}$ by substituting $S_{D}$ for $S_{\theta}$ and $\partial D / \partial N_{\text {qp }}$ for $\partial \theta / \partial N_{\mathrm{qp}}$. It can also predict the resonator sensitivity in the dissipation direction by using $\partial D / \partial N_{\mathrm{qp}}$ from the real part of Eq. (3) and the normalized voltage noise of an amplifier with noise temperature $T_{n}$ referenced to the center of the resonance loop,

$$
S_{D}^{\text {pred }}=\frac{2 k T_{n}}{P}\left(\frac{Q_{c}}{Q_{m}}\right)^{2}
$$

Combining Eqs. (3), (5), and (6) yields the predicted NEP based only on the device properties, shown as the red line in Fig. 3. This is a powerful tool for optimizing microstrip MKIDs for specific detector applications.

Figure 3 shows the calculated NEP for the resonator measured in Fig. 2. As expected, $\mathrm{NEP}_{\theta}$ is higher than $\mathrm{NEP}_{D}$ due to the contribution of TLSs to the phase noise. The measured $\mathrm{NEP}_{D}$, with a minimum below $5 \times 10^{-17} \mathrm{~W} \mathrm{~Hz}^{-1 / 2}$ between $10 \mathrm{~Hz}$ and $1 \mathrm{kHz}$, is a very good NEP competitive with many other low temperature detectors. There is a small unexplained rise in the $\mathrm{NEP}_{D}$ at very low frequencies, which is likely due to $1 /$ f gain fluctuations in the HEMT amplifier.

The ease and flexibility of fabrication combined with the good sensitivity makes microstrip MKIDs an extremely in-

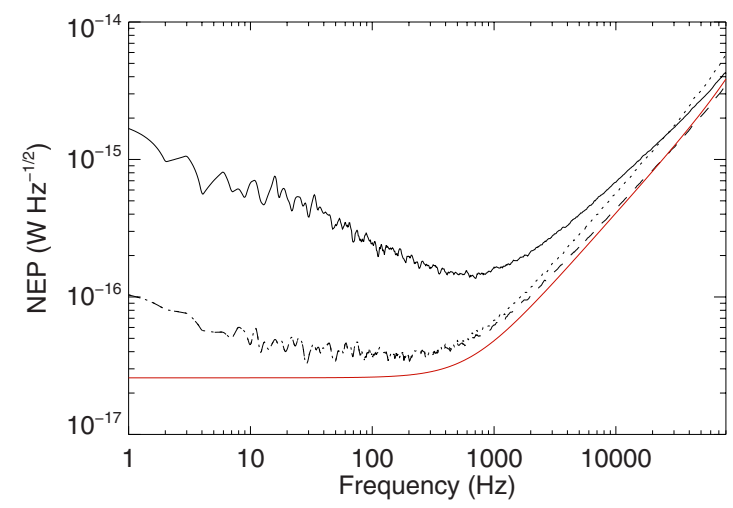

FIG. 3. (Color online) The NEP of the microstrip resonator. The solid line shows the NEP derived from phase shifts only, the dotted line is derived from dissipation data, and the dashed line is the optimal NEP using both dissipation and phase data (Ref. 14). The red line is the predicted NEP from Eqs. (3), (5), and (6) assuming there is $2 \mathrm{~dB}$ of loss between the device and a HEMT amplifier with $T_{n}=5.5 \mathrm{~K}$.

teresting device for future large detector arrays. Significantly higher performance in much smaller resonators can be achieved by using thinner films and superconductors like titanium that have long penetration depths.

This material is based upon work supported by the National Aeronautics and Space Administration under Grant No. NNH06ZDA001N-APRA2 issued through the Science Mission Directorate. The authors would like to thank John Martinis, Sunil Golwala, and Andrew Cleland for useful insights.

${ }^{1}$ P. K. Day, H. Leduc, B. A. Mazin, A. Vayonakis, and J. Zmuidzinas, Nature (London) 425, 817 (2003).

${ }^{2}$ P. R. Maloney, N. G. Czakon, P. K. Day, R. Duan, J. Gao, J. Glenn, S. Golwala, M. Hollister, H. G. LeDuc, B. Mazin, O. Noroozian, H. T. Nguyen, J. Sayers, J. Schlaerth, J. E. Vaillancourt, A. Vayonakis, P. Wilson, and J. Zmuidzinas, AIP Conf. Proc. 1185, 176 (2009).

${ }^{3}$ D. Moore, B. Mazin, S. Golwala, B. Bumble, J. Gao, B. Young, S. McHugh, P. Day, H. LeDuc, and J. Zmuidzinas, AIP Conf. Proc. 1185, 168 (2009).

${ }^{4}$ B. A. Mazin, B. Bumble, P. K. Day, M. E. Eckart, S. Golwala, J. Zmuidzinas, and F. A. Harrison, Appl. Phys. Lett. 89, 222507 (2006).

${ }^{5}$ A. Wallraff, D. Schuster, A. Blais, L. Frunzio, R. Huang, J. Majer, S. Kumar, S. Girvin, and R. Schoelkopf, Nature (London) 431, 162 (2004).

${ }^{6} J$. Martinis, K. Cooper, R. McDermott, M. Steffen, M. Ansmann, K. Osborn, K. Cicak, S. Oh, D. Pappas, R. Simmonds, and C. Yu, Phys. Rev. Lett. 95, 210503 (2005).

${ }^{7}$ C. A. Regal, J. D. Teufel, and K. W. Lehnert, Nat. Phys. 4, 555 (2008).

${ }^{8}$ G. J. Grabovskij, L. J. Swenson, O. Buisson, C. Hoffmann, A. Monfardini, and J. C. Villegier, Appl. Phys. Lett. 93, 134102 (2008).

${ }^{9}$ B. A. Mazin, Thesis from the Caltech Department of Physics, 2004.

${ }^{10}$ J. M. Martinis, Quantum Inf. Process. 8, 81 (2009).

${ }^{11}$ P. Day, H. Leduc, A. Goldin, T. Vayonakis, B. Mazin, S. Kumar, J. Gao, and J. Zmuidzinas, Nucl. Instrum. Methods Phys. Res. A 559, 561 (2006).

${ }^{12}$ J. Swihart, J. Appl. Phys. 32, 461 (1961).

${ }^{13}$ J. Pond, J. Claassen, and W. Carter, IEEE Trans Microwave Theory Tech. 35, 1256 (1987).

${ }^{14} \mathrm{~J}$. Gao, Thesis from the Caltech Department of Physics, 2008.

${ }^{15}$ A. D. O'Connell, M. Ansmann, R. C. Bialczak, M. Hofheinz, N. Katz, E. Lucero, C. McKenney, M. Neeley, H. Wang, E. M. Weig, A. N. Cleland, and J. M. Martinis, Appl. Phys. Lett. 92, 112903 (2008).

${ }^{16}$ J. Gao, M. Daal, A. Vayonakis, S. Kumar, J. Zmuidzinas, B. Sadoulet, B A. Mazin, P. K. Day, and H. G. Leduc, Appl. Phys. Lett. 92, 152505 (2008).

${ }^{17}$ J. Gao, M. Daal, J. M. Martinis, A. Vayonakis, J. Zmuidzinas, B. Sadoulet, B. A. Mazin, P. K. Day, and H. G. Leduc, Appl. Phys. Lett. 92, 212504 (2008).

${ }^{18}$ H. Wang, M. Hofheinz, J. Wenner, M. Ansmann, R. C. Bialczak, M. Lenander, E. Lucero, M. Neeley, A. D. O'Connell, D. Sank, M. Weides, A. N. Cleland, and J. M. Martinis, Appl. Phys. Lett. 95, 233508 (2009). 